\title{
INTRINSIC VOLUMES OF INSCRIBED RANDOM POLYTOPES IN SMOOTH CONVEX BODIES
}

\author{
I. BÁRÁNY,* Alfréd Rényi Institute of Mathematics and University College London \\ F. FODOR, ${ }^{* *}$ University of Szeged and University of Calgary \\ V. VÍGH, ${ }^{* * *}$ University of Szeged
}

\begin{abstract}
Let $K$ be a $d$-dimensional convex body with a twice continuously differentiable boundary and everywhere positive Gauss-Kronecker curvature. Denote by $K_{n}$ the convex hull of $n$ points chosen randomly and independently from $K$ according to the uniform distribution. Matching lower and upper bounds are obtained for the orders of magnitude of the variances of the $s$ th intrinsic volumes $V_{s}\left(K_{n}\right)$ of $K_{n}$ for $s \in\{1, \ldots, d\}$. Furthermore, strong laws of large numbers are proved for the intrinsic volumes of $K_{n}$. The essential tools are the economic cap covering theorem of Bárány and Larman, and the Efron-Stein jackknife inequality.
\end{abstract}

Keywords: Convex body; intrinsic volume; uniform random polytope; variance; strong law of large numbers

2010 Mathematics Subject Classification: Primary 52A22; 60D05

\section{Notation}

We will work in $d$-dimensional Euclidean space $\mathbb{R}^{d}$, with origin $o$, scalar product $\langle\cdot, \cdot\rangle$, and induced norm $\|\cdot\|$. The dimension $d$ will be fixed throughout the paper. We will not distinguish between the Euclidean space and the underlying vector space, and we will use the words point and vector interchangeably, as we need them. Points of $\mathbb{R}^{d}$ are denoted by small-case roman letters, and sets by capitals. For reals, we use either Greek letters or small-case letters. Let $B^{j}$ denote the $j$-dimensional ball of radius 1 centered at the origin, $S^{j-1}$ denote the boundary of $B^{j}$, and $\kappa_{j}$ denote the $j$-dimensional volume (Lebesgue measure) $\lambda_{j}\left(B^{j}\right)$ of $B^{j}$. We will also use the simple notation $V(\cdot)$ for the $d$-dimensional volume when no confusion is possible. Note that any point $x \in \partial B^{j}=S^{j-1}$ can be considered as a point of the boundary of $B^{j}$ and also as an outer normal to $B^{j}$ at the point $x$. For a point set $T \subset \mathbb{R}^{d}$, we denote the convex hull of $T$ by conv $T$ or simply by [T]. A compact convex set $K$ with nonempty interior is called a convex body.

\footnotetext{
Received 17 June 2009.

* Postal address: Department of Mathematics, University College London, Gower Street, London, WC1E 6BT, UK. Email address: barany@ renyi.hu

Supported by Hungarian OTKA grant 60427.

** Postal address: Department of Geometry, University of Szeged, Aradi vértanúk tere 1, H-6720 Szeged, Hungary.

Email address: fodorf@math.u-szeged.hu

Supported by Hungarian OTKA grants 68398 and 75016, and by the János Bolyai Research Scholarship of the Hungarian Academy of Sciences.

*** Postal address: Bolyai Institute, University of Szeged, Aradi vértanúk tere 1, H-6720 Szeged, Hungary.

Email address: vigvik@math.u-szeged.hu

Supported by Hungarian OTKA grant 75016.
} 
The intrinsic volumes $V_{s}(K), s=0, \ldots, d$, of a convex body $K$ can be introduced as coefficients of the Steiner formula

$$
V\left(K+\varepsilon B^{d}\right)=\sum_{s=0}^{d} \varepsilon^{d-s} \kappa_{d-s} V_{s}(K)
$$

where $K+\varepsilon B^{d}$ is the Minkowski sum of $K$ and $\varepsilon B^{d}$ of radius $\varepsilon \geq 0$. In particular, $V_{d}$ is the volume functional, $V_{0}(K)=1, V_{1}$ is proportional to the mean width, and $V_{d-1}$ is a multiple of the surface area. For more information on intrinsic volumes, see the monographs by Schneider [16], and Schneider and Weil [17]. To avoid confusion, sometimes we use $\lambda_{s}$ for the $s$-dimensional volume (in particular, $V_{d}=\lambda_{d}$ ).

For a convex body $K$ in $\mathbb{R}^{d}$, we say that $\partial K$ is $C_{+}^{k}$ for some $k \geq 2$ if $\partial K$ is a $C^{k}$ manifold and its Gaussian curvature is positive everywhere. For a convex body $K$ with $C^{2}$ boundary and $x \in \partial K$, we use $\sigma_{j}(x)$ for the $j$ th normalized elementary symmetric function of the principal curvatures of $\partial K$ at $x$. In particular, $\sigma_{d-1}(x)$ is the Gaussian curvature.

We integrate on $G(d, s)$, the Grassmannian manifold of $s$-dimensional linear subspaces of $\mathbb{R}^{d}$. The normalized (and unique) Haar measure on $G(d, s)$ is denoted by $v_{s}$ (for details, see [17, pp. 575-596]). If $L \in G(d, s)$ and $T \subset \mathbb{R}^{d}$, then we write $T \mid L$ for the orthogonal projection of $T$ onto $L$. We use $\mathbf{1}(\cdot)$ for the indicator function of a set. As usual, $\mathrm{E}(\cdot)$ and $\operatorname{var}(\cdot)$ stand for expectation and variance of a random variable. The notation ' $\ll$ ', ' $\gg$ ' and ' $\approx$ ' are used in the following sense. If $f(n), g(n): \mathbb{N} \rightarrow \mathbb{R}$ are two functions then we write $f \ll g$ if there exist a constant $\gamma$ and a positive number $n_{0}$ such that we have $f(n)<\gamma g(n)$ for all $n>n_{0}$. Furthermore, $f \approx g$ if $g \ll f \ll g$. If $n$ is a positive integer then $[n]$ denotes the set $\{1, \ldots, n\}$. We write $\left(\begin{array}{c}{[n]} \\ k\end{array}\right)$ for the set of all $k$-element subsets of $[n]$.

\section{History and results}

In this paper we consider the following probability model. Let $K$ be a $d$-dimensional convex body. Select the points $x_{1}, \ldots, x_{n}$ randomly and independently from $K$ according to the uniform probability distribution. The density of the uniform distribution with respect to the Lebesgue measure is the function with the constant value $\lambda_{d}(K)^{-1}$. The convex hull $K_{n}:=\left[x_{1}, \ldots, x_{n}\right]$ is a (uniform) random polytope inscribed in $K$. For a convex body $K$, the expectation $\mathrm{E}_{n}\left(V_{s}\right)$ of the $s$ th intrinsic volume of $K_{n}$ tends to $V_{s}(K)$ as $n$ tends to $\infty$, and the shape of the boundary of $K$ determines the asymptotic behavior of the random variable $V_{s}(K)-\mathrm{E}_{n}\left(V_{s}\right)$. In this paper, we prove matching lower and upper bounds for the order of magnitude of the variance of $V_{s}\left(K_{n}\right)$ for convex bodies with $C_{+}^{2}$ boundary. The upper bound on the variance will imply a strong law of large numbers for $V_{s}\left(K_{n}\right)$.

Much effort has been devoted to investigating the properties of various geometric functionals associated with uniform random polytopes. An up-to-date survey about the current state of this field can be found in the paper by Bárány [4], the book by Schneider and Weil [17, pp. 299-328], and also in the survey by Weil and Wieacker [20] from 1993. Here we only wish to give a brief outline of results that are directly connected with our results.

In particular, the following asymptotic formula is known about the expectation of intrinsic volumes $V_{s}(K), s=1, \ldots, d$. If the boundary $\partial K$ of $K$ is $C_{+}^{2}$ then

$$
\lim _{n \rightarrow \infty}\left(\frac{n}{V(K)}\right)^{2 /(d+1)}\left[V_{s}(K)-\mathrm{E} V_{s}\left(K_{n}\right)\right]=c_{d, s} \int_{\partial K} \sigma_{d-1}(x)^{1 /(d+1)} \sigma_{d-s}(x) \mathrm{d} x,
$$


with a constant $c_{d, s}>0$ depending only on $d$ and $s$. Formula (1) is due to Bárány [2] if $K$ has a $C_{+}^{3}$ boundary, and to Reitzner [14] if $K$ has a $C_{+}^{2}$ boundary.

Until quite recently, very little had been known about the variance of intrinsic volumes of uniform random polytopes. In 1993, in the survey paper by Weil and Wieacker [20], the authors state that 'the determination of the variance, for instance, is a major open problem'. Küfer [12] obtained the first result in this direction; he proved the upper bound $O\left(n^{-(d+3) /(d+1)}\right)$ for the variance of the missed volume for the $d$-dimensional unit ball. A major breakthrough was achieved by Reitzner [13], who proved that, for a convex body $K$ with $C_{+}^{2}$ boundary,

$$
\operatorname{var} V\left(K_{n}\right) \leq c(K) n^{-(d+3) /(d+1)},
$$

where the constant $c(K)$ depends on $K$ and the dimension only. The proof of Reitzner's result rests on the jackknife inequality of Efron and Stein [10], [11], which we also use in our argument. Böröczky et al. [9] obtained an upper bound of the same order of magnitude as in (2) for the mean width variance of a uniform random polytope for the case when the mother body has a rolling ball. Bárány and Reitzner [7] established an upper bound for the case of volume when $K$ is a polytope. More precisely, they proved that

$$
\operatorname{var} V\left(K_{n}\right) \leq c(K) \frac{1}{n^{2}}(\log n)^{d-1},
$$

where the constant $c(K)$ depends on $K$ and the dimension only.

The above upper bounds imply strong laws of large numbers for the corresponding functionals.

In [15] Reitzner proved matching lower bounds for the variance of the volume functional for convex bodies with $C_{+}^{2}$ boundary, that is,

$$
\operatorname{var} V\left(K_{n}\right) \geq c(K) n^{-(d+3) /(d+1)} .
$$

These lower bounds were extended by Bárány and Reitzner [7] to every convex body in the form

$$
\operatorname{var} V\left(K_{n}\right) \gg \frac{1}{n} V\left(K\left(\frac{1}{n}\right)\right),
$$

where $K(1 / n)$ is the wet part of $K$ with parameter $1 / n$; see Section 3 for details. The only known lower bound for the variance of an intrinsic volume of a uniform random polytope other than that of the volume is due to Böröczky et al. [9]. They established a lower bound with the order of magnitude $n^{-(d+3) /(d+1)}$ for the mean width of uniform random polytopes for the case when the mother body has a rolling ball.

The variance of the random variable $f_{s}\left(K_{n}\right)$, which is the number of $s$-dimensional faces of $K_{n}(s=0,1, \ldots, d-1)$, can be estimated using the above methods as shown in [7] and [13]. Very recently, Schreiber and Yukich [18] determined the variance of $f_{0}\left(K_{n}\right)$ asymptotically when $K$ is the unit ball, a significant breakthrough. Hopefully, their methods can work for all $f_{s}\left(K_{n}\right)$ and $V_{s}\left(K_{n}\right)$ as well.

In this paper we determine the order of magnitude of var $V_{s}\left(K_{n}\right)$ when $K=B^{d}$, the unit ball.

Theorem 1. Let $B^{d} \subset \mathbb{R}^{d}$ be the d-dimensional unit ball. Let $B_{n}^{d}$ be the convex hull of $n$ independent random points chosen from $B^{d}$ according to the uniform probability distribution. Then, for $s=1, \ldots, d$,

$$
\operatorname{var} V_{s}\left(B_{n}^{d}\right) \approx n^{-(d+3) /(d+1)} \text { as } n \rightarrow \infty .
$$


The proof of Theorem 1 can be extended to smooth convex bodies with $C_{+}^{2}$ boundary. All techniques used in the argument for the unit ball apply, with minor variations, to the case of smooth convex bodies. We give a brief outline of how this can be achieved in Section 6 .

Theorem 2. Let $K \subset \mathbb{R}^{d}$ be a convex body with $C_{+}^{2}$ boundary. Let $K_{n}$ be the convex hull of $n$ independent random points chosen from $K$ according to the uniform probability distribution. Then, for $s=1, \ldots, d$,

$$
\operatorname{var} V_{s}\left(K_{n}\right) \approx n^{-(d+3) /(d+1)} \quad \text { as } n \rightarrow \infty .
$$

The upper bound for the variance of the intrinsic volumes implies a strong law of large numbers via standard arguments. Thus, we obtain the following result.

Theorem 3. Let $K \subset \mathbb{R}^{d}$ be a convex body with $C_{+}^{2}$ boundary, and let $K_{n}$ be the convex hull of $n$ independent random points from $K$ chosen according to the uniform distribution. Then, for $s=1, \ldots, d$,

$$
\lim _{n \rightarrow \infty}\left(V_{s}(K)-V_{s}\left(K_{n}\right)\right) n^{2 /(d+1)}=c_{d, s} V(K)^{2 /(d+1)} \int_{\partial K} \sigma_{d-1}(x)^{1 /(d+1)} \sigma_{d-s}(x) \mathrm{d} x
$$

with probability 1 .

The lower bound on the variance can be used to prove the central limit theorem (CLT) for the random variable $V_{s}\left(\Pi_{n}\right)$. Here $\Pi_{n}$, the Poisson random polytope, is similar to the random polytope $K_{n}$, just for the Poisson polytope; the number of random points chosen from $K$ is a Poisson-distributed random variable with mean $n$. The method of proving the CLT for this case was introduced by Reitzner [15] and extended in [8]. It works, with more or less straightforward modifications, for the case of $V_{s}\left(\Pi_{n}\right)$ when $K$ is either the unit ball or a $C_{+}^{2}$ convex body. The actual proof is long, technical, and tedious, and does not use significant new ideas; therefore, it is omitted. Transferring the CLT from the Poisson polytope to the usual random polytope is often not so simple and was carried out, for $V\left(K_{n}\right)$ and $f_{s}\left(K_{n}\right)$, by $\mathrm{Vu}$ [19] for smooth convex bodies and by Bárány and Reitzner [8] for polytopes using different methods. The same transference for the mixed volumes will, most likely, require some new method.

\section{Tools}

In this section we describe two statements that will be used in our proof, and we will prove a lemma that will be a useful tool for both the lower and upper estimates of the variance.

If $K$ is a convex body then a cap of $K$ is a set $C=K \cap H_{+}$, where $H_{+}$is a closed half-space. We define the function $v: K \rightarrow \mathbb{R}$ as

$$
v(x):=\min \left\{\lambda_{d}\left(K \cap H_{+}\right) \mid x \in H_{+} \text {and } H_{+} \text {is a closed half-space }\right\} .
$$

The set $K(t)=K(v \leq t)=\{x \in K \mid v(x) \leq t\}$ is called the wet part of $K$ with parameter $t>0$. The remaining part of $K$, namely, $K(v \geq t)=\{x \in K \mid v(x) \geq t\}$, is the floating body of $K$ with parameter $t>0$. We can easily verify that if $K$ is a ball then $\lambda_{d}(K(t)) \approx t^{(d+1) / 2}$.

The following theorem of Bárány and Larman [6] and Bárány [1] plays a central role in our proof.

Theorem 4. (Economic cap covering.) Assume that $K$ is a convex body with unit volume, and let $0<t<t_{0}=(2 d)^{-2 d}$. Then there are caps $C_{1}, \ldots, C_{m}$ and pairwise disjoint convex sets $C_{1}^{\prime}, \ldots, C_{m}^{\prime}$ such that $C_{i}^{\prime} \subset C_{i}$ for each $i$, and

(i) $\bigcup_{1}^{m} C_{i}^{\prime} \subset K(t) \subset \bigcup_{1}^{m} C_{i}$, 
(ii) $V\left(C_{i}^{\prime}\right) \gg t$ and $V\left(C_{i}\right) \ll t$ for each $i$,

(iii) for each cap $C$ with $C \cap K(v>t)=\varnothing$, there is a $C_{i}$ containing $C$.

An immediate consequence of this theorem is that $m t \ll \lambda_{d}(K(t)) \ll m t$. For more details and for further references on the economical cap covering theorem, see [3] and [6].

Our second major tool is the Efron-Stein jackknife inequality (see [10], [11], and [13]). If $K_{n}$ denotes the random polytope inscribed in a convex body $K$ as above, then the original Efron-Stein theorem readily implies that

$$
\operatorname{var}\left(V_{s}\left(K_{n}\right)\right) \leq(n+1) \mathrm{E}\left(V_{s}\left(K_{n+1}\right)-V_{s}\left(K_{n}\right)\right)^{2} .
$$

Finally, we need a simple statement on the measure of special linear subspaces of $\mathbb{R}^{d}$. Assume that $z \in S^{d-1}$ and $A \in G(d, s)$ are given. Their angle, $L(z, A)$, is defined as the minimum of the angles $\angle(z, x)$ for all $x \in A$.

Lemma 1. For fixed $z \in S^{d-1}$ and small $\alpha>0, v_{s}\{A \in G(d, s) \mid \angle(z, A) \leq \alpha\} \approx \alpha^{d-s}$.

Proof. Let $L \in G(d, s-1)$ be a subspace in the orthogonal complement $z^{\perp}$ of the vector $z$. For every $e \in L^{\perp} \cap S^{d-1}$ with $\angle(e, z) \leq \alpha$, the subspace $A=\operatorname{linspan}(L \cup\{e\})$ makes an angle at most $\alpha$ with $z$. Also, conversely, every such subspace $A \in G(d, s)$ can be written in this form. It is not hard to see that the $v_{s}$-measure of this set is approximately equal to $\alpha^{d-s}$.

\section{Proof of the lower bound in Theorem 1}

The idea of the proof of the lower bound is similar to that presented in [9] and [15], namely, we define small independent caps, and we show that the variance is 'large' in each cap. From the properties of the variance, the required estimate will follow.

We will use Kubota's formula (see [17, p. 222]) to represent intrinsic volumes as mean projections:

$$
V_{s}(K)=c(d, s) \int_{G(d, s)} \lambda_{s}(K \mid L) v_{s}(\mathrm{~d} L),
$$

where $c(d, s)$ is a constant depending only on $d$ and $s$.

For $x \in S^{d-1}$ and $t \in(0,1)$, we define $H(x, t)=\left\{z \in \mathbb{R}^{d} \mid\langle z, x\rangle=1-t\right\}$ and we write $x_{t}=(1-t) x$. Let $C(x, t)$ be the smaller cap cut off from $B^{d}$ by $H(x, t)$. We call $x$ the center of this cap. Clearly, $B(x, t)=H(x, t) \cap B^{d}$ is a $(d-1)$-dimensional ball centered at the point $x_{t}$. The radius of $B(x, t)$ is $\sqrt{t(2-t)}$, showing that

$$
\left(x_{t}+\sqrt{t} B^{d}\right) \cap H(x, t) \subset H(x, t) \cap B^{d} \subset\left(x_{t}+\sqrt{2 t} B^{d}\right) \cap H(x, t) .
$$

This implies that, for all $t \in(0,1)$, we have

$$
C(x, t) \subset x+2 \sqrt{t} B^{d} .
$$

In fact, we will work with $t$ very close to 0 (see (11), below), and all inequalities with ' $\ll$ ' below are meant with $t \rightarrow 0$.

Next we inscribe a regular $(d-1)$-simplex into $B(x, t)$, whose vertices are the points $w_{1}, w_{2}, \ldots, w_{d} \in \partial B(x, t)$. It follows from (6) that the simplex $\left[w_{1}, \ldots, w_{d}\right]$ contains the $(d-1)$-ball of radius $\sqrt{t} / d$ centered at the point $x_{t}$. Set $w_{0}=x$. Then $\Delta=\left[w_{0}, w_{1}, \ldots, w_{d}\right]$ is a $d$-dimensional simplex inscribed in $C(x, t)$. 
For all $j=0,1, \ldots, d$, we define

$$
\Delta_{j}=\Delta_{j}(x, t)=w_{j}+\frac{1}{4 d}\left(\left[w_{0}, w_{1}, \ldots, w_{d}\right]-w_{j}\right) .
$$

Here $\Delta_{j}$ is a homothetic copy of $\Delta$ with center $w_{j}$ and factor $1 /(4 d)$. It readily follows from (6) that $V\left(\Delta_{j}(x, t)\right) \approx t^{(d+1) / 2}$. Choose a point $z_{j}$ in each $\Delta_{j}(x, t)$, and define

$$
\Sigma_{1}(x, t)=S^{d-1} \cap\left(x+\frac{\sqrt{t}}{8} B^{d}\right)
$$

and

$$
\Sigma_{2}(x, t)=S^{d-1} \cap\left(x+2 d \sqrt{t} B^{d}\right) .
$$

Set $\Delta(z)=\left[z_{0}, \ldots, z_{d}\right]$, and write $N$ for the cone of outer normals to $\Delta(z)$ at vertex $z_{0}$. We claim that

$$
\Sigma_{1}(x, t) \subset S^{d-1} \cap N \subset \Sigma_{2}(x, t) .
$$

To prove (8), pick an arbitrary $v \in S^{d-1}$ such that $\langle v, x\rangle=0$. From the definition of $\Delta_{j}$ and (6), we obtain

$$
\frac{\sqrt{t}}{2 d} \leq h_{\Delta(z)}(v)-\left\langle z_{0}, v\right\rangle \leq 2 \sqrt{t}
$$

where $h_{\Delta(z)}(\cdot)$ is the support function of $\Delta(z)$. Similarly,

$$
\frac{t}{2} \leq\left\langle z_{0}, x\right\rangle-h_{\Delta(z)}(x) \leq t .
$$

From these we deduce that the 'extremal' element $u$ of the normal cone $N$ in the direction $v(u=\langle u, x\rangle x+\langle u, v\rangle v)$ satisfies

$$
\frac{\sqrt{t}}{4} \leq \tan (\angle(u, x)) \leq 2 d \sqrt{t}
$$

and so claim (8) follows.

Relation (8) can be dualized:

$$
\Sigma_{2}^{*}(x, t) \subset\left\{\lambda\left(y-z_{0}\right) \mid \lambda \geq 0, y \in\left[z_{0}, z_{1}, \ldots, z_{d}\right]\right\} \subset \Sigma_{1}^{*}(x, t),
$$

where $\Sigma_{j}^{*}(x, t)=\left\{y \mid\langle y, u\rangle \leq 0\right.$ for all $\left.u \in \Sigma_{j}(x, t)\right\}$ is the usual dual cone of $\Sigma_{j}$. Note that (8) also implies that there exists an absolute constant $\gamma$ such that

$$
B^{d} \backslash C(x, \gamma t) \subset z_{0}+\Sigma_{2}^{*}(x, t) .
$$

Now fix $x, t$, and $z_{j} \in \Delta_{j}(x, t)$ for $j=1, \ldots, d$. We write $F=\left[z_{1}, \ldots, z_{d}\right]$. Define the function $\hat{V}_{s}: \Delta_{0}(x, t) \rightarrow \mathbb{R}$ as follows:

$$
\hat{V}_{s}\left(z_{0}\right)=\int_{L \in G(d, s), L \cap \Sigma_{2} \neq \varnothing} \lambda_{s}\left(\left[z_{0}, F\right] \mid L\right) v_{s}(\mathrm{~d} L) .
$$

Here $\hat{V}_{s}$ clearly depends on $F$; if we want to emphasize this dependence then we write $\hat{V}_{s}\left(z_{0} ; F\right)$.

Lemma 2. If $Z$ is a random point chosen uniformly from $\Delta_{0}(x, t)$ then

$$
\operatorname{var} \hat{V}_{s}(Z) \gg t^{d+1} \text {. }
$$


Proof. Let $w$ be the centroid of the facet of $\Delta_{0}(x, t)$ opposite to $x$, and let $w_{1}=\frac{2}{3} x+\frac{1}{3} w$ and $w_{2}=\frac{1}{3} x+\frac{2}{3} w$. In addition, define

$$
\Psi_{1}=\left(w_{1}-\Sigma_{2}^{*}(x, t)\right) \cap \Delta_{0}(x, t), \quad \Psi_{2}=\left(w_{2}+\Sigma_{2}^{*}(x, t)\right) \cap \Delta_{0}(x, t) .
$$

In particular, there exists some constant $\gamma_{0}>0$ such that

$$
V\left(\Psi_{j}\right) \geq \gamma_{0} V\left(\Delta_{0}(x, t)\right),
$$

and, for any $Z_{1} \in \Psi_{1}$ and $Z_{2} \in \Psi_{2}$, we have $\left[Z_{2}, z_{1}, \ldots, z_{d}\right] \subset\left[Z_{1}, z_{1}, \ldots, z_{d}\right]$.

Fix $L \in G(d, s)$ such that $L \cap \Sigma_{2} \neq \varnothing$, and choose an orthonormal basis $e_{1}, \ldots, e_{s}$ in $L$ such that $e_{1} \in L \cap \Sigma_{2}$.

Consider the closed (positive) half-space given by $w_{2}$ and $e_{1}$, i.e. $H_{1}^{+}=\left\{y \mid\left\langle y, e_{1}\right\rangle \geq\right.$ $\left.\left\langle w_{2}, e_{1}\right\rangle\right\}$, and the set $G=H_{1}^{+} \cap\left(Z_{1}+\Sigma_{2}^{*}(x, t)\right)$. Clearly, $G \subset\left[F, Z_{1}\right]$ and $G \cap\left[F, Z_{2}\right] \subset$ $\left\{w_{2}\right\}$. From these, it follows that

$$
\lambda_{s}\left(\left[F, Z_{1}\right] \mid L\right)-\lambda_{s}\left(\left[F, Z_{2}\right] \mid L\right) \geq \lambda_{s}(G \mid L) .
$$

We can see that

$$
\lambda_{s}(G \mid L) \gg t \sqrt{t}^{s-1}=t^{(s+1) / 2}
$$

hence,

$$
\hat{V}_{s}\left(Z_{1}\right)-\hat{V}_{s}\left(Z_{2}\right) \gg t^{(s+1) / 2} v_{s}\left(\left\{L \mid L \cap \Sigma_{2} \neq \varnothing\right\}\right) .
$$

By the definition of $\Sigma_{2}, L \cap \Sigma_{2} \neq \varnothing$ is equivalent to $L \cap(x+2 d \sqrt{t} B) \neq \varnothing$. Lemma 1 yields

$$
\hat{V}_{s}\left(Z_{1}\right)-\hat{V}_{s}\left(Z_{2}\right) \gg t^{(d+1) / 2} \text {. }
$$

Finally, we obtain

$$
\begin{aligned}
\operatorname{var} \hat{V}_{s}(Z) & =\frac{1}{2} \mathrm{E}\left(\hat{V}_{s}\left(Z_{1}\right)-\hat{V}_{s}\left(Z_{2}\right)\right)^{2} \\
& \geq \frac{1}{2} \mathrm{E}\left(\left(\hat{V}_{s}\left(Z_{1}\right)-\hat{V}_{s}\left(Z_{2}\right)\right)^{2} \mathbf{1}\left(Z_{1} \in \Psi_{1}, Z_{2} \in \Psi_{2}\right)\right) \\
& \gg t^{d+1} \mathrm{E}\left(\mathbf{1}\left(Z_{1} \in \Psi_{1}, Z_{2} \in \Psi_{2}\right)\right) \\
& \gg t^{d+1}
\end{aligned}
$$

where the last inequality follows from (10).

It is sufficient to prove the lower bound for large enough $n$. We fix

$$
t_{n}=n^{-2 /(d+1)}
$$

and, hence, $V\left(C\left(x, t_{n}\right)\right) \approx 1 / n$ for all $x \in S^{d-1}$. We choose a maximal family of points $y_{1}, \ldots, y_{m} \in S^{d-1}$ such that, for $i \neq j$, we have

$$
\left\|y_{i}-y_{j}\right\| \geq 2 \sqrt{\gamma} \sqrt{t_{n}} .
$$

This condition implies that the caps $C\left(y_{j}, \gamma T_{n}\right)(j \in[m])$ are disjoint. We can see that

$$
m \gg n^{(d-1) /(d+1)} \text {. }
$$

For each $j \in[m]$, we construct the simplex $\Delta\left(y_{j}, t_{n}\right)$ in the cap $C\left(y_{j}, t_{n}\right)$ and, for each $i=0,1, \ldots, d$, we construct the corresponding small simplices $\Delta_{i}\left(y_{j}, t_{n}\right)$. For $j \in[m]$, 
let $A_{j}$ denote the event that each $\Delta_{i}\left(y_{j}, t_{n}\right), i=0, \ldots, d$, contains exactly one random point out of $x_{1}, \ldots, x_{n}$, and $C\left(y_{j}, \gamma t_{n}\right)$ contains no other random point. We note that the definition of $\Delta_{i},(7)$, and (9) yield, for $i=0, \ldots, d$,

$$
V\left(\Delta_{i}\left(y_{j}, t_{n}\right)\right) \gg \frac{1}{n} \quad \text { and } \quad V\left(C\left(y_{j}, \gamma t_{n}\right)\right) \ll \frac{1}{n} .
$$

Thus, for $j=1, \ldots, m$, we have

$$
\mathrm{P}\left(A_{j}\right) \gg\left(\begin{array}{c}
n \\
d+1
\end{array}\right)\left(\frac{1}{n}\right)^{d+1}\left(1-\frac{1}{n}\right)^{n-d-1} \gg 1 .
$$

If $A_{j}$ holds then we write $Z_{j}$ to denote the random point in $\Delta_{0}\left(y_{j}, t_{n}\right)$, and $F_{j}$ to denote the convex hull of the random points in $\Delta_{i}\left(y_{j}, t_{n}\right)$ for $i=1, \ldots, d$. If $J \subset[m]$ and $A_{j}$ holds for all $j \in J$, then the random variables $\hat{V}_{s}\left(Z_{j} ; F_{j}\right), j \in J$, are independent according to (9).

We next introduce the $\sigma$-algebra $\mathcal{F}$ that keeps track of everything except the location of $Z_{j} \in \Delta_{0}\left(y_{j}, t_{n}\right)$ for which $A_{j}$ occurs. We decompose the variance by conditioning on $\mathcal{F}$ :

$$
\operatorname{var} V_{s}\left(K_{n}\right)=\mathrm{E} \operatorname{var}\left(V_{s}\left(K_{n}\right) \mid \mathcal{F}\right)+\operatorname{var} \mathrm{E}\left(V_{s}\left(K_{n}\right) \mid \mathcal{F}\right) \geq \mathrm{E}\left(\operatorname{var} V_{s}\left(K_{n}\right) \mid \mathcal{F}\right) .
$$

The independence structure mentioned above implies that

$$
\operatorname{var}\left(V_{S}\left(K_{n}\right) \mid \mathcal{F}\right)=\sum_{\mathbf{1}\left(A_{j}\right)=1} \operatorname{var}_{Z_{j}} V_{s}\left(K_{n}\right)=\sum_{\mathbf{1}\left(A_{j}\right)=1} \operatorname{var}_{Z_{j}} \hat{V}_{s}\left(Z_{j} ; F_{j}\right),
$$

where the variance is taken with respect to the random variable $Z_{j} \in \Delta_{0}\left(y_{j}, t_{n}\right)$, and we sum over all $j=1, \ldots, m$ with $\mathbf{1}\left(A_{j}\right)=1$. Combining this with Lemma 2, (11), (12), and (13) implies that

$$
\operatorname{var} V_{S}\left(K_{n}\right) \gg \mathrm{E}\left(\sum_{\mathbf{1}\left(A_{j}\right)=1} t_{n}^{d+1}\right) \gg n^{-2} \mathrm{E}\left(\sum_{j=1}^{m} I\left(A_{j}\right)\right) \gg n^{-2} m \gg n^{-(d+3) /(d+1)}
$$

\section{Proof of the upper bound in Theorem 1}

Now $K=B^{d}$ is the unit ball and $K_{n}$ is the corresponding random polytope.

Let $T_{n}$ be the event that the floating body $K(v \geq V(K)(c \ln n) / n)$ is contained in $K_{n}$. Here $c=c_{d}$ is a large constant to be specified soon. We write $T_{n}^{\mathrm{c}}$ for the complement of $T_{n}$. We are going to use the main result of [5] which states that there is a constant $\delta$ depending only on $d$ such that $T_{n}^{\mathrm{c}}$ occurs with probability $n^{-\delta c}$.

We use the Efron-Stein jackknife inequality (4):

$$
\begin{aligned}
\operatorname{var}\left(V_{s}\left(K_{n}\right)\right) & \ll n \mathrm{E}\left(V_{s}\left(K_{n+1}\right)-V_{s}\left(K_{n}\right)\right)^{2} \\
& =n \mathrm{E}\left(\left(V_{s}\left(K_{n+1}\right)-V_{s}\left(K_{n}\right)\right)^{2} \mathbf{1}\left(T_{n}\right)\right)+n \mathrm{E}\left(\left(V_{s}\left(K_{n+1}\right)-V_{s}\left(K_{n}\right)\right)^{2} \mathbf{1}\left(T_{n}^{\mathrm{c}}\right)\right) .
\end{aligned}
$$

The second term here is very small if the constant $c$ is chosen large enough because $\left(V_{s}\left(K_{n+1}\right)-\right.$ $\left.V_{s}\left(K_{n}\right)\right)^{2} \leq V_{s}\left(K_{n+1}\right)^{2} \leq V_{s}(K)^{2}$ and $\mathrm{E}\left(\mathbf{1}\left(T_{n}^{\mathrm{c}}\right)\right) \leq n^{-\delta c}$. We choose $c=c_{d}$ so large that the second term is smaller than the lower bound in Theorem 1 proved in the previous section. So we 
concentrate on the first term:

$$
\begin{aligned}
\operatorname{var}\left(V_{s}\left(K_{n}\right)\right) \ll & \ll \operatorname{E}\left(\left(V_{s}\left(K_{n+1}\right)-V_{s}\left(K_{n}\right)\right)^{2} \mathbf{1}\left(T_{n}\right)\right) \\
\ll & \mathbb{E}\left(\left(\int_{G(d, s)} \lambda_{s}\left(K_{n+1} \backslash K_{n} \mid A\right) v_{s}(\mathrm{~d} A)\right)\right. \\
& \left.\quad \times\left(\int_{G(d, s)} \lambda_{s}\left(K_{n+1} \backslash K_{n} \mid B\right) v_{s}(\mathrm{~d} B) \mathbf{1}\left(T_{n}\right)\right)\right) \\
\ll & \quad \int_{G(d, s)} \int_{G(d, s)} \lambda_{s}\left(K_{n+1} \backslash K_{n} \mid A\right) \\
\times & \lambda_{s}\left(K_{n+1} \backslash K_{n} \mid B\right) \mathbf{1}\left(T_{n}\right) v_{s}(\mathrm{~d} A) v_{s}(\mathrm{~d} B) .
\end{aligned}
$$

Note that the set $\left(K_{n+1} \backslash K_{n}\right) \mid A$ is either empty (if $x_{n+1}\left|A \in K_{n}\right| A$ ) or it is the union of several internally disjoint simplices which are the convex hull of $x_{n+1} \mid A$ and those facets of $K_{n} \mid A$ that can be seen from $x_{n+1} \mid A$. For the index set $I=\left\{i_{1}, \ldots, i_{s}\right\} \subset\{1, \ldots, n\}$, let $F_{I}=\left[x_{i_{1}}, \ldots, x_{i_{s}}\right]$, which is an $(s-1)$-dimensional simplex with probability 1 . Clearly, $F_{I} \mid A$ is also an $(s-1)$-simplex with probability 1 . The affine hull of $F_{I}$ is denoted by aff $F_{I}$ and similarly the affine hull of $F_{I} \mid A$ is denoted by aff $\left(F_{I} \mid A\right)$. Furthermore, let $H_{0}\left(F_{I}, A\right)$ be the closed half-space (in $\mathbb{R}^{d}$ ) delimited by the hyperplane $A^{\perp}+$ aff $F_{I}$ that contains $o$, and let $H_{+}\left(F_{I}, A\right)$ be the other half-space. Similarly, we use $H_{0}\left(F_{I} \mid A\right)$ and $H_{+}\left(F_{I} \mid A\right)$ for the corresponding $s$-dimensional half-spaces in $A$. Now, we introduce the notation $\mathcal{F}(A)$ for the set of $\left((s-1)\right.$-dimensional) facets of $K_{n} \mid A$ that can be seen from $x_{n+1} \mid A$ :

$$
\mathcal{F}(A)=\left\{F_{I}\left|A: K_{n}\right| A \subset H_{0}\left(F_{I} \mid A\right), x_{n+1} \mid A \in H_{+}\left(F_{I} \mid A\right), I=\left\{i_{1}, \ldots, i_{s}\right\} \subset\{1, \ldots, n\}\right\} .
$$

Of course, $\mathcal{F}(A)$ depends on $x_{1}, \ldots, x_{n}$ and $x_{n+1}$ as well, but we suppress this dependence in the notation. We continue by estimating the right-hand side of (14) (denoted RHS $(14)$ ):

$$
\begin{aligned}
\operatorname{RHS}_{(14)} \leq & n \mathrm{E}\left[\int_{G(d, s)} \int_{G(d, s)} \lambda_{s}\left(K_{n+1} \backslash K_{n} \mid A\right) \lambda_{s}\left(K_{n+1} \backslash K_{n} \mid B\right) v_{s}(\mathrm{~d} A) v_{s}(\mathrm{~d} B) \mathbf{1}\left(T_{n}\right)\right] \\
\ll & \frac{n}{\kappa_{d}^{n+1}} \int_{K} \cdots \int_{K} \int_{G(d, s)} \int_{G(d, s)}\left(\sum_{F \in \mathcal{F}(A)} \lambda_{s}\left(\left[x_{n+1} \mid A, F\right]\right)\right) \\
& \times\left(\sum_{F^{\prime} \in \mathcal{F}(B)} \lambda_{s}\left(\left[x_{n+1} \mid B, F^{\prime}\right]\right) \mathbf{1}\left(T_{n}\right)\right) v_{s}(\mathrm{~d} A) v_{s}(\mathrm{~d} B) \mathrm{d} x_{1} \cdots \mathrm{d} x_{n+1} .
\end{aligned}
$$

By changing the order of integration and extending the integration over all index sets $I, J \in\left(\begin{array}{c}{[n]} \\ s\end{array}\right)$, we obtain

$$
\begin{aligned}
& \frac{n}{\kappa_{d}^{n+1}} \int_{G(d, s)} \int_{G(d, s)} \int_{K^{n+1}}\left(\sum_{I} \mathbf{1}\left(F_{I} \mid A \in \mathcal{F}(A)\right) \lambda_{s}\left(\left[F_{I}, x_{n+1}\right] \mid A\right)\right) \\
& \times\left(\sum_{J} \mathbf{1}\left(F_{J} \mid B \in \mathcal{F}(B)\right) \lambda_{s}\left(\left[F_{J}, x_{n+1}\right] \mid B\right) \mathbf{1}\left(T_{n}\right)\right) \mathrm{d} x_{1} \cdots \mathrm{d} x_{n+1} v_{s}(\mathrm{~d} A) v_{s}(\mathrm{~d} B) .
\end{aligned}
$$

We use the following notation. Let

$$
C_{S}(I, A)=H_{+}\left(F_{I} \mid A\right) \cap B^{d},
$$


which is, in fact, a subset of the unit ball in the subspace $A$ and

$$
C_{d}(I, A)=\left(H_{+}\left(F_{I} \mid A\right)+A^{\perp}\right) \cap B^{d} .
$$

For the volumes of these caps, we use $V_{s}(I, A)=\lambda_{s}\left(C_{s}(I, A)\right)$ and $V_{d}(I, A)=\lambda_{d}\left(C_{d}(I, A)\right)$. Now we estimate these integrals from above using the fact that the simplices $\left[F_{I}, x_{n+1}\right] \mid A$ and $\left[F_{J}, x_{n+1}\right] \mid B$ are contained in the associated caps $C_{s}(I, A)$ and $C_{S}(J, B)$, respectively. Hence,

$$
\begin{aligned}
(15) \ll & \frac{n}{\kappa_{d}^{n+1}} \int_{G(d, s)} \int_{G(d, s)} \sum_{I} \sum_{J} \int_{\left(B^{d}\right)^{n+1}} \mathbf{1}\left(F_{I} \mid A \in \mathcal{F}(A)\right) V_{s}(I, A) \\
& \times \mathbf{1}\left(F_{J} \mid B \in \mathcal{F}(B)\right) V_{S}(J, B) \mathbf{1}\left(T_{n}\right) \mathrm{d} x_{1} \cdots \mathrm{d} x_{n+1} v_{s}(\mathrm{~d} A) v_{s}(\mathrm{~d} B) .
\end{aligned}
$$

The summation extends over all $s$-tuples $I$ and $J$, so $I$ and $J$ may have nonempty intersection. If we fix the size of $I \cap J$ to be $k$, say, then the corresponding terms in the sum are clearly independent of the particular choice of $i_{1}, \ldots, i_{s}$ and $j_{1}, \ldots, j_{s}$. For any given $k \in\{0,1, \ldots, s\}$, let $I=\{1, \ldots, s\}$ and $J=\{s-k+1, \ldots, 2 s-k\}$, and set $F=\operatorname{conv}\left\{x_{i}: i \in I\right\}$ and $G=\operatorname{conv}\left\{x_{j}: j \in J\right\}$. Thus, $I$ and $J$, and, consequently, $F$ and $G$, depend on $k$, but this is not shown in the notation. We can estimate (16) from above:

$$
\begin{aligned}
(16) \leq & \frac{n}{\kappa_{d}^{n+1}} \sum_{k=0}^{s}\left(\begin{array}{l}
n \\
s
\end{array}\right)\left(\begin{array}{l}
s \\
k
\end{array}\right)\left(\begin{array}{l}
n-s \\
s-k
\end{array}\right) \int_{G(d, s)} \int_{G(d, s)} \int_{\left(B^{d}\right)^{n+1}} \mathbf{1}(F \mid A \in \mathcal{F}(A)) \\
& \times V_{s}(I, A) \mathbf{1}(G \mid B \in \mathcal{F}(B)) V_{s}(J, B) \mathbf{1}\left(T_{n}\right) \mathrm{d} x_{1} \cdots \mathrm{d} x_{n+1} v_{s}(\mathrm{~d} A) v_{s}(\mathrm{~d} B) .
\end{aligned}
$$

Since the integrand is symmetric, we may restrict summation to those pairs of $F$ and $G$ where $V_{s}(I, A) \geq V_{s}(J, B)$, or, equivalently, $V_{d}(I, A) \geq V_{d}(J, B)$, at the price of a factor 2 . Thus, we can estimate (17) from above:

$$
\begin{aligned}
(17) \leq & \sum_{k=0}^{s} \frac{2 n}{\kappa_{d}^{n+1}}\left(\begin{array}{l}
n \\
s
\end{array}\right)\left(\begin{array}{l}
s \\
k
\end{array}\right)\left(\begin{array}{l}
n-s \\
s-k
\end{array}\right) \int_{G(d, s)} \int_{G(d, s)} \int_{\left(B^{d}\right)^{n+1}} \mathbf{1}(F \mid A \in \mathcal{F}(A)) \\
& \times V_{s}(I, A) \mathbf{1}(G \mid B \in \mathcal{F}(B)) V_{s}(J, B) \mathbf{1}\left(V_{s}(I, A) \geq V_{s}(J, B)\right) \\
& \times \mathbf{1}\left(T_{n}\right) \mathrm{d} x_{1} \cdots \mathrm{d} x_{n+1} v_{s}(\mathrm{~d} A) v_{s}(\mathrm{~d} B) .
\end{aligned}
$$

Let $\Sigma_{k}$ denote the $k$ th term in this sum, $k=0, \ldots, s$. We are going to estimate $\Sigma_{k}$ for each fixed $k$.

We first remove $\mathbf{1}(G \mid B \in \mathcal{F}(B))$ from the integrand in $\Sigma_{k}$, which clearly increases the integral. We then multiply the integrand by $\mathbf{1}\left(C_{d}(I, A) \cap C_{d}(J, B) \neq \varnothing\right)$. This does not change the integral since the sets $C_{d}(I, A)$ and $C_{d}(J, B)$ have at least the point $x_{n+1}$ in common. Thus, we obtain

$$
\begin{aligned}
\Sigma_{k} \ll & \frac{n^{2 s-k+1}}{\kappa_{d}^{n+1}} \int_{G(d, s)} \int_{G(d, s)} \int_{\left(B^{d}\right)^{n+1}} \mathbf{1}(F \mid A \in \mathcal{F}(A)) V_{s}(I, A) \\
& \times \mathbf{1}\left(C_{d}(I, A) \cap C_{d}(J, B) \neq \varnothing\right) V_{s}(J, B) \mathbf{1}\left(V_{s}(I, A) \geq V_{s}(J, B)\right) \\
& \times \mathbf{1}\left(T_{n}\right) \mathrm{d} x_{1} \cdots \mathrm{d} x_{n+1} v_{s}(\mathrm{~d} A) v_{s}(\mathrm{~d} B) .
\end{aligned}
$$

Now, if $F \mid A \in \mathcal{F}(A)$ then $x_{2 s-k+1}, \ldots, x_{n}$ are all contained in $H_{0}(F, A)$ and $x_{n+1}$ is contained in $H_{+}(F, A)$ because, under condition $T_{n}, C_{d}(I, A)$ is the smaller cap cut off from $B^{d}$ 
by the hyperplane $A^{\perp}+$ aff $F$ and $o \in K_{n}$. We integrate with respect to $x_{2 s-k+1}, \ldots, x_{n}, x_{n+1}$, and the condition $T_{n}$ is replaced by the condition $W_{n}$ saying that $V_{d}(I, A) \leq \kappa_{d}(c \ln n) / n$ :

$$
\begin{aligned}
\Sigma_{k} \ll & \frac{n^{2 s-k+1}}{\kappa_{d}^{n+1}} \int_{G(d, s)} \int_{G(d, s)} \int_{\left(B^{d}\right)^{2 s-k}}\left(\kappa_{d}-V_{+}(F, A)\right)^{n-2 s+k} V_{d}(I, A) \\
& \times V_{s}(I, A) \mathbf{1}\left(C_{d}(I, A) \cap C_{d}(J, B) \neq \varnothing\right) V_{s}(J, B) \\
& \times \mathbf{1}\left(V_{d}(I, A) \geq V_{d}(J, B)\right) \mathbf{1}\left(W_{n}\right) \mathrm{d} x_{1} \cdots \mathrm{d} x_{2 s-k} v_{s}(\mathrm{~d} A) v_{s}(\mathrm{~d} B) \\
\ll & n^{2 s-k+1} \int_{G(d, s)} \int_{G(d, s)} \int_{\left(B^{d}\right)^{2 s-k}}\left(1-\frac{V_{d}(I, A)}{\kappa_{d}}\right)^{n-2 s+k} \\
& \times V_{d}(I, A) V_{s}(I, A) \mathbf{1}\left(C_{d}(I, A) \cap C_{d}(J, B) \neq \varnothing\right) V_{s}(J, B) \\
& \times \mathbf{1}\left(V_{d}(I, A) \geq V_{d}(J, B)\right) \mathbf{1}\left(W_{n}\right) \mathrm{d} x_{1} \cdots \mathrm{d} x_{2 s-k} v_{s}(\mathrm{~d} A) v_{s}(\mathrm{~d} B) .
\end{aligned}
$$

In the next step, we integrate with respect to the variables $x_{i}, i \in J$, that is, we estimate the following integral expression:

$$
\begin{gathered}
\int_{\left(B^{d}\right)^{s-k}} \mathbf{1}\left(C_{d}(I, A) \cap C_{d}(J, B) \neq \varnothing\right) \mathbf{1}\left(V_{d}(I, A) \geq V_{d}(J, B)\right) \\
\times V_{s}(J, B) \mathbf{1}\left(W_{n}\right) \mathrm{d} x_{s+1} \cdots \mathrm{d} x_{2 s-k} .
\end{gathered}
$$

Since we assume that $V_{d}(I, A) \geq V_{d}(J, B)$, the height of the cap $C_{d}(I, A)$ is at least that of $C_{d}(J, B)$. The condition $C_{d}(I, A) \cap C_{d}(J, B) \neq \varnothing$ implies that there is a constant $\beta$ such that $C_{d}(J, B)$ is contained in $\beta C_{d}(I, A)$, where $\beta C_{d}(I, A)$ is an enlarged homothetic copy of $C_{d}(I, A)$, where the center of homothety is $z \in \partial B^{d}$, the center of the cap $C_{d}(I, A)$ (cf. [3]). Thus,

$$
(19) \leq \beta^{d(s-k)} V_{d}(I, A)^{s-k} V_{s}(J, B) \ll V_{d}(I, A)^{s-k} V_{s}(I, A) .
$$

The conditions $C_{d}(I, A) \cap C_{d}(J, B) \neq \varnothing$ and $V_{d}(I, A) \geq V_{d}(J, B)$ can only be satisfied if the angle between the vector $z$ and the subspace $B, \angle(z, B)$, is not larger than $2 \alpha$, where $\alpha$ is the central angle of the cap $C_{d}(I, A)$. We can easily verify that

$$
\alpha \leq b_{d} V_{d}(I, A)^{1 /(d+1)},
$$

where $b_{d}$ is a constant depending only on $d$. Using this condition on the mutual positions of $z$ and $B$ together with (20), we obtain

$$
\begin{aligned}
(18) \ll n^{2 s-k+1} \int_{G(d, s)} \int_{G(d, s)} \int_{\left(B^{d}\right)^{s}} & \left(1-\frac{V_{d}(I, A)}{\kappa_{d}}\right)^{n-2 s+k} V_{d}(I, A)^{s-k+1} \\
& \times V_{s}(I, A)^{2} \mathbf{1}\left(L(z, B) \leq 2 b_{d} V_{d}(I, A)^{1 /(d+1)}\right) \\
& \times \mathbf{1}\left(W_{n}\right) \mathrm{d} x_{1} \cdots \mathrm{d} x_{s} v_{s}(\mathrm{~d} A) v_{s}(\mathrm{~d} B) .
\end{aligned}
$$

We now fix $A \in G(d, s)$ and estimate

$$
\int_{\left(B^{d}\right)^{s}}\left(1-\frac{V_{d}(I, A)}{\kappa_{d}}\right)^{n-2 s+k} V_{d}(I, A)^{s-k+1} V_{s}(I, A)^{2} \mathbf{1}\left(W_{n}\right) \mathrm{d} x_{1} \cdots \mathrm{d} x_{s} .
$$

We are going to use the economic cap covering theorem. Because of the condition $W_{n}$, every cap $C_{d}(I, A)$ has volume at most $\kappa_{d}(c \ln n) / n$. Let $h$ be a (positive) integer with $2^{-h} \leq(c \ln n) / n$. 
For each such $h$, let $\mathcal{M}_{h}$ be a collection of caps $\left\{C_{1}, \ldots, C_{m(h)}\right\}$ forming the economic cap covering of the wet part of $B^{s}=B^{d} \mid A$ with $t=\left(\kappa_{d} 2^{-h}\right)^{(s+1) /(d+1)}$ (we suppose that $n$ is so large that the theorem works). Each such cap $C_{i}$ is the projection of a $d$-dimensional cap $C_{i}(A)$ from $B^{d}$ to $A$. Since the heights of $C_{i}$ and $C_{i}(A)$ are equal, we have $\lambda_{d}\left(C_{i}(A)\right) \ll \kappa_{d} 2^{-h}$. Consider an arbitrary $\left(x_{1}, \ldots, x_{s}\right)$ with the corresponding $C_{d}(I, A)$ having volume at most $\kappa_{d}(c \ln n) / n$, and associate with $\left(x_{1}, \ldots, x_{s}\right)$ the maximal $h$ such that, for some $C_{i} \in \mathcal{M}_{h}$, $C_{s}(I, A) \subset C_{i}$. Such an $h$ clearly exists. It follows that

$$
V_{s}(I, A) \leq \lambda_{s}\left(C_{i}\right) \ll 2^{-h(s+1) /(d+1)}
$$

and

$$
V_{d}(I, A) \leq \lambda_{d}\left(C_{i}(A)\right) \ll 2^{-h}
$$

On the other hand, by the maximality of $h$,

$$
V_{s}(I, A) \geq\left(\kappa_{d} 2^{-(h+1)}\right)^{(s+1) /(d+1)},
$$

and, consequently,

$$
\frac{V_{d}(I, A)}{\kappa_{d}} \geq 2^{-(h+1)} .
$$

Now we integrate over $\left(B^{d}\right)^{s}$ under condition $W_{n}$ by integrating each $\left(x_{1}, \ldots, x_{s}\right)$ on its associated $C_{i}(A)$ or, more precisely, on $\left(C_{i}(A)\right)^{s}$. The integrand in (23) can be estimated as

$$
\begin{aligned}
(1- & \left.\frac{V_{d}(I, A)}{\kappa_{d}}\right)^{n-2 s+k} V_{d}(I, A)^{s-k+1} V_{s}(I, A)^{2} \\
& \ll\left(1-2^{-(h+1)}\right)^{n-2 s+k} 2^{-h(s-k+1)} 2^{-2 h(s+1) /(d+1)} .
\end{aligned}
$$

Thus, the integral on $\left(C_{i}(A)\right)^{s}\left(C_{i}(A) \in \mathcal{M}_{h}\right)$ is bounded by

$$
\begin{array}{r}
\exp \left(-(n-2 s+k) 2^{-h-1}\right) 2^{-h(s+k-1)} 2^{-2 h(s+1) /(d+1)}\left(V_{d}\left(C_{i}(A)\right)\right)^{s} \\
\ll \exp \left(-(n-2 s+k) 2^{-h-1}\right) 2^{-h(s+k-1)} 2^{-2 h(s+1) /(d+1)} 2^{-h s} .
\end{array}
$$

Now we return to (22). In order to estimate the integral, we still need the number of elements, $\left|\mathcal{M}_{h}\right|$, of $\mathcal{M}_{h}$. The volume of the wet part of $B^{s}$ with parameter $2^{-h(s+1) /(d+1)}$ is $\lambda_{s}\left(B^{s}\left(2^{-h(s+1) /(d+1)}\right)\right) \approx 2^{-2 h /(d+1)}($ the ' $\approx$ ' notation makes sense, since $h \rightarrow \infty$ as $n \rightarrow \infty)$. It readily follows that

$$
\left|\mathcal{M}_{h}\right| \ll \frac{2^{-2 h /(d+1)}}{2^{-h(s+1) /(d+1)}}=2^{h(s-1) /(d+1)} .
$$

Keeping in mind the condition $\angle(z, B) \leq 2 b_{d} V_{d}(I, A)^{1 /(d+1)}$ and applying Lemma 1 and (24), we obtain, with $h_{0}=\lfloor c \ln n / n\rfloor$,

$$
\begin{aligned}
& \int_{G(d, s)} \int_{\left(B^{d}\right)^{s}}\left(1-\frac{V_{d}(I, A)}{\kappa_{d}}\right)^{n-2 s+k} V_{d}(I, A)^{s-k+1} V_{s}(I, A)^{2} \\
& \times \mathbf{1}\left(\angle(z, B) \leq 2 b_{d} V_{d}(I, A)^{1 /(d+1)}\right) \mathrm{d} x_{1} \cdots \mathrm{d} x_{s} v_{s}(\mathrm{~d} B) \\
& \ll \sum_{h=h_{0}}^{\infty} \exp \left(-(n-2 s+k) 2^{-h-1}\right) 2^{-h(s+k-1)} 2^{-2 h(s+1) /(d+1)} 2^{-h s} \\
& \times\left|\mathcal{M}_{h}\right| v_{s}\left(\left\{B \mid \angle(z, B)<2 b_{d} 2^{-h /(d+1)}\right\}\right)
\end{aligned}
$$




$$
\begin{aligned}
& \ll \sum_{h=h_{0}}^{\infty} \exp \left(-(n-2 s+k) 2^{-h-1}\right) \\
& \quad \times 2^{-h(s+k-1)} 2^{-2 h(s+1) /(d+1)} 2^{-h s} 2^{h(s-1) /(d+1)} 2^{-h(d-s) /(d+1)} \\
& =\sum_{h=h_{0}}^{\infty} \exp \left(-(n-2 s+k) 2^{-h+1}\right) 2^{-h[(2 s-k+1)+(d+3) /(d+1)]} .
\end{aligned}
$$

Now, we divide the sum in (24) into two parts. First, let $h_{1}$ be defined by

$$
2^{-h_{1}} \leq \frac{1}{n}<2^{-h_{1}+1}
$$

Since in this case $\left.\exp \left(-(n-2 s+k) 2^{-h-1}\right)\right)$ is smaller than 1 , it follows that

$$
\begin{aligned}
\sum_{h=h_{1}}^{\infty} \exp \left(-(n-2 s+k) 2^{-h-1}\right) 2^{-h[(2 s-k+1)+(d+3) /(d+1)]} & \leq \sum_{h=h_{1}}^{\infty} 2^{-h[(2 s-k+1)+(d+3) /(d+1)]} \\
& \ll n^{-2 s+k-1} n^{-(d+3) /(d+1)} .
\end{aligned}
$$

For the other part, when $h_{0} \leq h<h_{1}$, we let $\ell=h_{1}-h$. Then $\ell$ runs from 1 to $\ell_{1}=h_{1}-h_{0}$. Thus,

$$
\begin{aligned}
& \sum_{h=h_{0}}^{h_{1}-1} \exp \left(-(n-2 s+k) 2^{-h-1}\right) 2^{-h[(2 s-k+1)+(d+3) /(d+1)]} \\
& \quad \leq \sum_{\ell=1}^{\ell_{1}} \exp \left(-(n-2 s+k) 2^{-h_{1}+\ell-1}\right) 2^{-\left(h_{1}-\ell\right)[(2 s-k+1)+(d+3) /(d+1)]} \\
& \quad \ll \sum_{\ell=1}^{\ell_{1}} \exp \left(-(n-2 s+k) 2^{-h_{1}+\ell-1}\right) n^{-(2 s-k+1)} 2^{\ell(2 s-k+1)} n^{-(d+3) /(d+1)} 2^{\ell(d+3) /(d+1)} \\
& \quad \ll n^{-(2 s-k+1)} n^{-(d+3) /(d+1)} \sum_{\ell=1}^{\infty} \exp \left(-2^{\ell}\right) 2^{\ell[(2 s-k+1)+(d+3) /(d+1)]} \\
& \ll n^{-(2 s-k+1)} n^{-(d+3) /(d+1)} \sum_{j=1}^{\infty} \exp (-j) j^{4 d} \\
& \ll n^{-(2 s-k+1)} n^{-(d+3) /(d+1)} .
\end{aligned}
$$

Now, substituting (25) and (26) into (22), we obtain

$$
\Sigma_{k} \ll n^{2 s-k+1} \int_{G(d, s)} n^{-(2 s-k+1)} n^{-(d+3) /(d+1)} v_{s}(\mathrm{~d} A) \ll n^{-(d+3) /(d+1)} .
$$

Summing this for all $k=0, \ldots, s$ proves the upper bound in Theorem 2 .

\section{Outline of the proof of Theorem 2}

In this section we give a brief outline of how to modify the proof of Theorem 1 so that it applies to convex bodies with $C_{+}^{2}$ boundary. We chose to give the detailed proof only for the 
unit ball, because all the major ideas appear in that case and it is naturally easier to follow. The proof in the general case uses the same tools as the proof for the unit ball combined with some well-known estimates about convex bodies. We make only a few remarks about the proof and leave the details for the interested reader.

Since $K$ is compact, there exist a global upper bound $\gamma$ and a global lower bound $\Gamma$ on the principal curvatures of $\partial K$. We also know that, for every $x \in \partial K$, there exists a unique outer unit normal $u_{x}$ to $K$ at the point $x$. We define the cap $C(x, t)$ such that it is cut off by the hyperplane $H(x, t):=\left\{y \mid\left\langle y, u_{x}\right\rangle=\left\langle x, u_{x}\right\rangle-t\right\}$. It readily follows that (6) remains true in the following form:

$$
\left(x_{t}+\gamma_{1} \sqrt{t} B^{d}\right) \cap H(x, t) \subset H(x, t) \cap K \subset\left(x_{t}+\gamma_{2} \sqrt{t} B^{d}\right) \cap H(x, t),
$$

where the constants $\gamma_{1}$ and $\gamma_{2}$ depend on $\gamma$ and $\Gamma$. From these estimates, it follows that the simplices used in the proof can be defined in the same way as in the case of the unit ball, and they have the same size, approximately $t^{(d+1) / 2}$. We also need a slight modification of the definitions of $\Sigma_{1}$ and $\Sigma_{2}$ :

$$
\Sigma_{1}(x, t)=S^{d-1} \cap\left(u_{x}+\frac{\sqrt{\gamma t}}{8} B^{d}\right)
$$

and

$$
\Sigma_{2}(x, t)=S \cap\left(u_{x}+2 d \sqrt{\Gamma t} B^{d}\right) .
$$

From this point, the steps of the proof can be followed without complications. For the details of a similar argument, see [7] or [9].

For the proof of the upper bound, we require that all projected images of $K$ also have a $C_{+}^{2}$ boundary; furthermore, we can choose $\gamma$ and $\Gamma$ in such a way that they are not only upper and lower bounds of the principal curvatures of $K$ but also for all ( $s$-dimensional) projections of $K$. It follows from these facts that the volume of a cap of height $t$ is approximately equal to $t^{(i+1) / 2}$, where $i$ is the dimension of the cap (in the proof $d$ or $s$, respectively). From (27), it follows that (21) remains true. We note that we did not really need the economic cap covering theorem in the case of the ball; however, in the general case we make a full use of it. Naturally, the stated equalities on the volumes of the caps are not true any more, but the existence of $\gamma$ and $\Gamma$ implies that they hold with ' $\approx$ '. The calculations completing the proof can be done precisely the same way as for the unit ball.

\section{Proof of Theorem 3}

We are going to show that the asymptotic formula (1) and Theorem 2 yield the strong law of large numbers for $V_{s}\left(K_{n}\right)$ by standard arguments.

We deduce by Chebyshev's inequality that

$$
\begin{aligned}
\mathrm{P}\left(\left|V_{S}(K)-V_{s}\left(K_{n}\right)-\mathrm{E}\left(V_{s}(K)-V_{s}\left(K_{n}\right)\right)\right| n^{2 /(d+1)} \geq \varepsilon\right) & \leq \varepsilon^{-2} n^{4 /(d+1)} \operatorname{var} V_{s}\left(K_{n}\right) \\
& \ll n^{-(d-1) /(d+1)} .
\end{aligned}
$$

Since the sum $\sum_{k=2}^{\infty} n_{k}^{-(d-1) /(d+1)}$ is finite for $n_{k}=k^{4}$, the sum of the probabilities

$$
\mathrm{P}\left(\left|V_{s}(K)-V_{s}\left(K_{n_{k}}\right)-\mathrm{E}\left(V_{s}(K)-V_{s}\left(K_{n_{k}}\right)\right)\right| n_{k}^{2 /(d+1)} \geq \varepsilon\right) \quad \text { for } k \geq 2
$$


is finite as well. Therefore, the Borel-Cantelli lemma and the asymptotic formula (1) yield

$$
\lim _{k \rightarrow \infty}\left(V_{s}(K)-V_{s}\left(K_{n_{k}}\right)\right) n_{k}^{2 /(d+1)}=c_{d, s} V(K)^{2 /(d+1)} \int_{\partial K} \sigma_{d-1}(x)^{1 /(d+1)} \sigma_{d-s}(x) \mathrm{d} x
$$

with probability 1 . Now, $V_{s}(K)-V_{s}\left(K_{n}\right)$ is decreasing, and, hence,

$$
\left(V_{s}(K)-\left(V_{s}\left(K_{n_{k-1}}\right)\right) n_{k-1}^{2 /(d+1)} \leq\left(V_{s}(K)-V_{s}\left(K_{n}\right)\right) n^{2 /(d+1)} \leq\left(V_{s}(K)-V_{s}\left(K_{n_{k}}\right)\right) n_{k}^{2 /(d+1)}\right.
$$

holds for $n_{k-1} \leq n \leq n_{k}$. As $\lim _{k \rightarrow \infty} n_{k} /\left(n_{k-1}\right)=1$, the subsequence limit theorem yields Theorem 3.

\section{References}

[1] BÁrÁNy, I. (1989). Intrinsic volumes and $f$-vectors of random polytopes. Math. Ann. 285, 671-699.

[2] BÁrÁNY, I. (1992). Random polytopes in smooth convex bodies. Mathematika 39, 81-92. (Correction: 51 (2004), 31.)

[3] BÁRÁNy, I. (2004). Random polytopes, convex bodies, and approximation. In Stochastic Geometry (Lecture Notes Math. 1892), eds A. Baddeley et al., Springer, Berlin.

[4] BáráNy, I. (2008). Random points and lattice points in convex bodies. Bull. Amer. Math. Soc. 45, 339-365.

[5] BÁrÁNy, I. ANd DAlla, L. (1997). Few points to generate a random polytope. Mathematika 44, 325-331.

[6] Bárány, I. and Larman, D. G. (1988). Convex bodies, economic cap coverings, random polytopes. Mathematika 35, 274-291.

[7] Bárány, I. and Reitzner, M. (2010). On the variance of random polytopes. To appear in Adv. Math.

[8] Bárány, I. and Reitzner, M. (2010). Poisson polytopes. Ann. Prob. 38, 1507-1531.

[9] Böröczky, K. J., Fodor, F., Reitzner, M. And VíGH, V. (2009). Mean width of random polytopes in a reasonably smooth convex body. J. Multivariate Anal. 100, 2287-2295.

[10] Efron, B. (1965). The convex hull of a random set of points. Biometrika 52, 331-343.

[11] Efron, B. And Stein, C. (1981). The jackknife estimate of variance. Ann. Statist. 9, 586-596.

[12] Küfer, K.-H. (1994). On the approximation of a ball by random polytopes. Adv. Appl. Prob. 26, 876-892.

[13] Reitzner, M. (2003). Random polytopes and the Efron-Stein jackknife inequality. Ann. Prob. 31, $2136-2166$.

[14] Reitzner, M. (2004). Stochastic approximation of smooth convex bodies. Mathematika 51, 11-29.

[15] Reitzner, M. (2005). Central limit theorems for random polytopes. Prob. Theory Relat. Fields 133, $483-507$.

[16] Schneider, R. (1993). Convex Bodies: the Brunn-Minkowski Theory. Cambridge University Press.

[17] SchneIder, R. AND WeIL, W. (2008). Stochastic and Integral Geometry. Springer, Berlin.

[18] SCHREIBER, T. AND YUKICH, J. E. (2008). Variance asymptotics and central limit theorems for generalized growth processes with applications to convex hulls and maximal points. Ann. Prob. 36, 363-396.

[19] Vu, V. (2006). Central limit theorems for random polytopes in a smooth convex set. Adv. Math. 207, $221-243$.

[20] Weil, W. and Wieacker, J. A. (1993). Stochastic geometry. In Handbook of Convex Geometry, eds P. M. Gruber and J. M. Wills, North-Holland, Amsterdam, pp. 1391-1438. 